\title{
Comprehensive gene expression analysis of the DNA (cytosine-5) methyltransferase family in rice (Oryza sativa L.)
}

\author{
F. Ahmad ${ }^{1}$, X. Huang ${ }^{1}$,H.X. Lan ${ }^{1}$, T. Huma ${ }^{2}$, Y.M. Bao ${ }^{1}$, J. Huang ${ }^{1}$ and \\ H.S. Zhang ${ }^{1}$ \\ ${ }^{1}$ State Key Laboratory of Crop Genetics and Germplasm Enhancement, \\ Nanjing Agricultural University, Nanjing, China \\ ${ }^{2}$ Reproductive Neuroendocrinology Laboratory, \\ Department of Animal Sciences, Faculty of Biological Sciences, \\ Quaid-i-Azam University, Islamabad, Pakistan \\ Corresponding authors: J. Huang / H.S. Zhang \\ E-mail: huangji@njau.edu.cn / hszhang@njau.edu.cn
}

Genet. Mol. Res. 13 (3): 5159-5172 (2014)

Received February 21, 2014

Accepted June 4, 2014

Published July 7, 2014

DOI http://dx.doi.org/10.4238/2014.July.7.9

\begin{abstract}
Cytosine DNA methylation is a conserved epigenetic regulatory mechanism in both plants and animals. DNA methyltransferases (DNA MTases) not only initiate (de novo) but also maintain the process of DNA methylation. Here, we characterized the genome-wide expression profiles of 10 cytosine DNA MTase genes belonging to 4 subfamilies, MET1, CMT, DNMT2, and DRM, in rice. Tissue-specific gene expression analysis showed that all family members varied widely in their expression and specificities and might be involved in some basic metabolic pathways. Similarly, the expression of all rice cytosine DNA MTase genes was not regulated by plant hormones except OsDRMIa and OsDRMIb, which were downregulated by jasmonic acid. The transcription level of 10 genes in rice shoots and roots was also measured under salt and osmotic stress. Meanwhile, quantitative polymerase chain reaction data of the japonica
\end{abstract}


and indica rice cultivars revealed that there is large variation in the expression activities of all genes. The results provide a foundation to further explore the roles of DNA MTases and the epigenetic regulation of abiotic stress responses in rice.

Key words: Rice; DNA methylation; DNA methyltransferases; Salt stress; Osmotic stress

\section{INTRODUCTION}

Rice not only feeds half of the world population but is also useful for basic molecular and genetic studies because of its relatively small genome size. A large portion of the rice crop may be destroyed in the field because of biotic and abiotic stresses. Salt and osmotic (dehydration) stresses are 2 major abiotic stresses for agricultural production (Zhu, 2002). In order to resist the environmental hazards, plants activate various molecular and biological processes (Rausher, 2001). In addition to various regulatory processes, DNA methylation, an epigenetic regulatory process, also plays a key role in the modulation of these stresses (Chinnusamy and Zhu, 2009). In plants, DNA methylation is a covalently attached methyl $\left(\mathrm{CH}_{3}\right)$ group at the 5-position of cytosine, yielding 5-methylcytosine. Although this cytosine can be methylated in any sequence context in plants but methylation at $\mathrm{CpG}$ and $\mathrm{CpNpG}$ sequences is very common (Chan et al., 2005).

In fact, the mechanism of DNA methylation is under the control of specific enzymes called DNA methyltransferases (DNA MTases). The number of cytosine DNA MTases varies among different organisms. In fungi, protozoa, and insects, a single methylase performs the methylation, while more than 10 are involved in higher plants like rice (Sharma et al., 2009). To understand the mechanism of DNA methylation under environmental stresses in rice, we analyzed the activities of MTases under 2 major abiotic stresses (salt and osmotic) separately and by dividing the plant into root and shoot. In rice, there are 4 basic subfamilies of DNA MTases to begin (de novo methylation) and to continue (maintenance methylation) DNA methylation: DNA methyltransferase 1 (MET1), DNA methyltransferase 2 (DNMT2), chromomethyltransferase (CMT), and domains rearranged methyltransferase (DRM) (Henikoff and Comai, 1998; Finnegan and Kovac, 2000; Cao and Südhof, 2001). OsMET1 and OsCMT3 mainly function in maintaining DNA hypermethylation at CG and CHG sites (where H is A, $\mathrm{T}$, or $\mathrm{C}$ ) during DNA duplication. In contrast, DRM2 is involved in de novo DNA methylation at CHH sites directed by RNA (Chan et al., 2006). In eukaryotes, the DNA methylation process is vital for normal growth and development; a mutation in the $\mathrm{CpG}$ maintenance MTase (DNMTs) is embryonic lethal in both plants and animals (Li et al., 1993; Rice et al., 2003; Xiao et al., 2006).

The epigenetic pedals of gene expression are not well understood, but evidence indicates that patterns of DNA methylation are important. In plants, specific DNA MTases (METI, $D R M$, and CMT) are essential for the creation of such patterns. Altered patterns of DNA methylation (as observed in $d d m 1$ or met1 mutants) can be transmissible in many generations, even following backcrossing to wild-type plants (Reinders et al., 2009; Colomé-Tatché et al., 2012). Meanwhile, genes of different DNA MTases are expressed differentially under different conditions. For example, ZmMET1 was downregulated by cold stress in maize (Ohba et 
al., 2000). In Arabidopsis, the transcription level of MET1 is at least 10,000 times more than that of MET11 (Sieńko et al., 2007).

Here, we analyzed the quantitative reverse transcription polymerase chain reaction (qRT-PCR)-based expression of cytosine DNA MTases in rice shoots and roots under salt and osmotic stress. Similarly, a comparative expression analysis of all MTases between the japonica and indica cultivars was also conducted.

\section{MATERIAL AND METHODS}

\section{Plant material and growth conditions}

Three rice (Oryza sativa) cultivars, Zonghua11 (ZH11), Nipponbare (japonica), and 93-11 (indica) were used in this experiment. Briefly, first we used ZH11 to construct an expression profile of DNA MTase genes in roots and shoots. The seeds were sterilized with $0.1 \%$ $\mathrm{HgCl}_{2}$ and then were transferred to a growth chamber at a fixed temperature of $28^{\circ} \mathrm{C}$. Daily, we supplied Yoshida's nutrient solution to the seedling as described previously (Yoshida, 1973; Rana et al., 2012). For the expression analysis in different tissues, ZH11 plants were grown under natural conditions, and samples were unruffled at the booting stage. Finally, Nipponbare and 93-11 were grown and treated as mentioned above. The samples were collected from 2-week-old seedlings of the 3 cultivars.

\section{Application of stresses and preparation of samples}

After 14 days of fixed conditions, the seedlings of all 3 cultivars were subjected to salt and osmotic stress, and samples were prepared. For the application of salt stress, we transferred the seedlings to a separate tray containing $150 \mathrm{mM} \mathrm{NaCl}$. For the osmotic stress, we used $20 \%$ polyethylene glycol 6000 (providing an osmotic potential of $-0.54 \mathrm{MPa}$ ) in another tray. Seedlings in a third tray were maintained in normal Yoshida's nutrient solution as a control. The seedlings were sampled after $12 \mathrm{~h}$ of treatments and immediately stored at $-80^{\circ} \mathrm{C}$.

\section{Identification and phylogenetic analysis of 10 DNA MTase genes}

A total of 10 genes encoding cytosine DNA MTases in the rice genome (accession Nos. AK242475, AK112062, AK109728, AK111502, AK108034, AK111461, AK063482, AK065147, AK065147, and AK065147) were identified as reported previously (Sharma et al., 2009). Individually, each accession number was used to form a rice proteome database profile to confirm each gene. We also used the Rice Genome Annotation Project (http://rice.plantbiology.msu.edu/) and chromatin database (http://www.chromdb.org). Later, we performed phylogenetic analysis of all cytosine DNA MTase domain-containing proteins from different plant species with MEGA5 using the neighbor-joining method (Tamura et al., 2011).

\section{RNA extraction and RT-PCR}

Total RNA was extracted from control and stressed (salt and osmotic) rice seedlings and from all other samples using Trizol reagent (Invitrogen, USA) according to the manufac- 
turer protocol. For the removal of genomic DNA, we used DNase1 (Takara, Japan) at $37^{\circ} \mathrm{C}$ for $15 \mathrm{~m}$. Total RNA purified $(2 \mu \mathrm{g})$ was used for the synthesis of cDNA using an RT-PCR system (Promega, USA) according to the manufacturer protocol.

\section{Quantitative real-time PCR}

An ABI-7500 Fast Real-Time PCR (Applied Biosystems, USA) with an optical 96well plate was used with SYBR Green Real-time PCR Master Mix (Toyobo, Japan) according to manufacturer instructions. The QuantPrime designing tool (Arvidsson et al., 2008) was used to design all primers (Table 1). For the proper quantification of MTase genes, we used $2 \mu \mathrm{g}$ of total RNA for the process of reverse transcription (Promega, USA) according to the protocol. Using the comparative $\mathrm{CT}\left(\Delta \Delta^{\mathrm{CT}}\right)$ method as described previously (Jain et al., 2006), the fold change in cDNA (target gene) relative to the reference gene (18s rRNA) was analyzed. The relative quantification relates the PCR signal of the target transcript in stress-treated rice seedlings to that of rice seedlings grown under normal conditions. The expression level of all MTase genes in roots, shoots, and different organs of rice plants and in the japonica and indica rice cultivars was analyzed as reported previously (Huang et al., 2008).

\section{Statistical analysis}

One-way analysis of variance and Bonferroni's multiple comparison test using the GraphPad5 software (GraphPad Software, Inc., USA) were used for the statistical analysis. P $<0.05$ was considered to be statistically significant.

\section{RESULTS}

\section{Characterization and phylogeny of MTases in rice}

Different classes of cytosine DNA MTases (CMT, DNMT2, MET, and DRM) were characterized, and a phylogenetic tree was constructed using the neighbor-joining method (Figure 1). MTases have a catalytic domain with conserved motifs that are arranged in a specific order. Because of this characteristic, it is believed that the cytosine DNA MTases originated from a common ancestor. Correspondingly, in eukaryotes, this domain is allied with $\mathrm{N}$ terminal extensions having a variable number of domains conserved across different MTases (Ponger and $\mathrm{Li}, 2005$ ). This proper structural arrangement promotes interactions between MTases and an array of other cellular proteins (Lindroth et al., 2004).

In plants and especially in rice, 4 subfamilies of cytosine-5 MTases have been identified (Pavlopoulou and Kossida, 2009; Sharma et al., 2009): CMT, DNMT2, MET1, and DRM (Finnegan et al., 1996; Henikoff and Comai, 1998). After identifying and analyzing a complete set of 10 de novo and maintenance MTase genes in rice, we aligned the protein of these specific MTases with a set of proteins from Zea mays and Nicotiana tabacum using the CLUSTALX software (Thompson et al., 1997) and generated a bootstrapped tree (Figure 1). The phylogenetic tree clearly divided plant MTases into 4 subfamilies.

In rice, there is a single putative OsDnmt 2 gene that has $99 \%$ similarity with the corresponding gene of maize. Similarly, 3 OsCMT genes were identified in rice that showed 


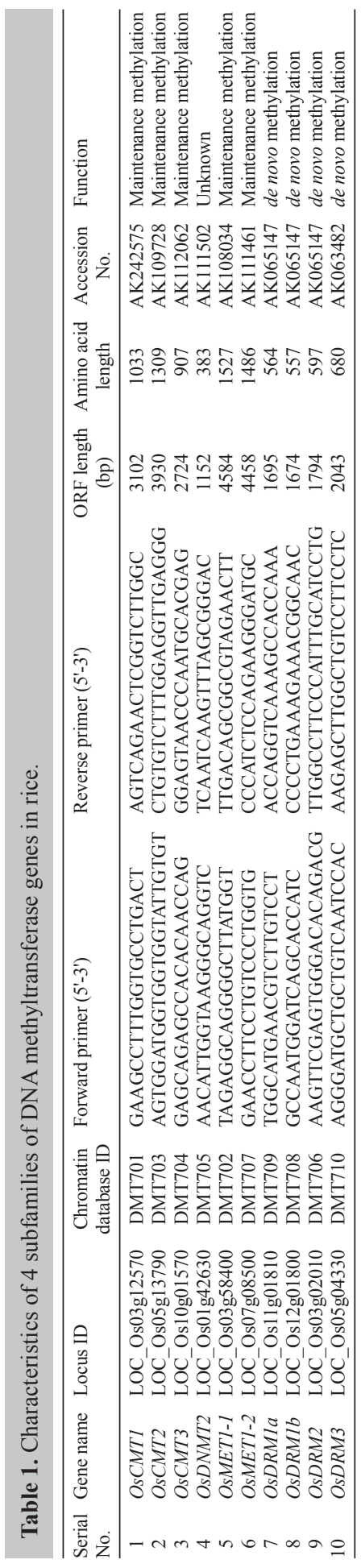




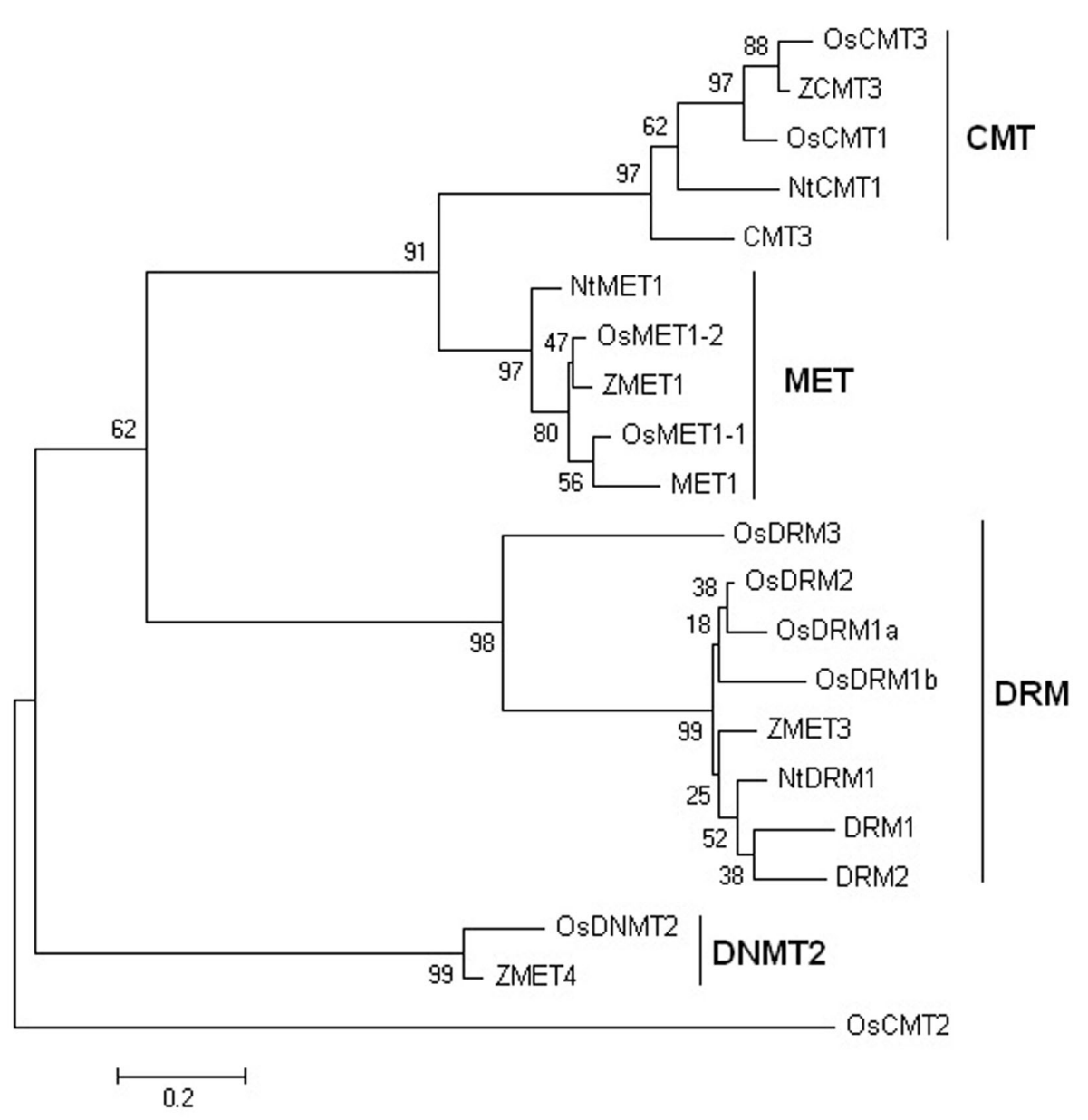

Figure 1. Phylogenetic relationship of plant DNA methyltransferase (MTase) proteins. Total protein sequences of rice, maize, and tobacco were downloaded from the chromatin database. The MSU accession numbers for rice DNA MTase proteins are shown in Table 1, and those of other proteins are: tobacco NtCMT1 (AB032538), NtMET1 (AB030726), and NtDRM1 (AB087883); and maize ZCMT3 (AAK15805.1), ZMET1 (AF063403), ZMET3 (AAF68437.1), and ZMET4 (AAK40306.1). ClustalX was used for multiple sequence alignment, and a phylogenetic tree was constructed using the neighbor-joining method with the MEGA5 software.

significant similarity to the corresponding genes of maize and tobacco. The 3 CMT genes (OsCMT1-3), used for their resemblance to the corresponding protein in Arabidopsis and other plant species. The OsCMT1-3 were characterized by the presence of a BAH domain in the N-terminal half and a preserved chromodomain inserted between motif I and motif IV (Sharma et al., 2009). OsCMT2 deviated from the other 2 proteins (Figure 1) by an additional stretch of 193 amino acids at the beginning of the BAH domain in the amino terminal region. The chromodomain of rice CMTs has a preserved aromatic amino acid, tyrosine, at position 412 and tryptophan at position 409. Similarly, a phenylalanine was observed to be at position 382 in all plant proteins in place of a tyrosine at the same position in the animal protein. 
Two already reported MET1 genes (MET1-1 and MET1-2) (Teerawanichpan et al., 2004; Yamauchi et al., 2008) that encode a conserved BAH domain and a C-terminal catalytic domain similar to the animal Dnmt1 protein were also used for phylogenetic analysis. Both of the genes (MET1-1 and MET1-2) in rice showed a high degree of similarity to the corresponding genes in maize and tobacco. All DRM proteins (DRM1a, DRM1b, DRM2, and DRM3) in rice had preserved motifs in the MTase domain: motif I and X were juxtaposed, demonstrating a match with DRMs of maize and tobacco. All MTase genes in rice showed roughly equal genomic distribution excluding the OsDRMIa and OsDRMIb genes, which were located in segmental duplicated regions of chromosomes 11 and 12, although the other genes were located in unique regions that are definite to each chromosome. In addition, all rice genes in the phylogenetic tree have at least 1 ortholog in maize, suggesting that the expansion of plant MTase genes occurred at least before the divergence of monocot species.

\section{Tissue-specific expression profiles of DNA MTases}

Different members of the MTase family have been noted for their differential expression patterns in different plant organs and tissues. The normalized expression of all 10 DNA MTase genes was determined in various tissues [seedling, stem, node, blade, sheath, young (y)-panicle, and flowering (f)-panicle] of rice. The quantitative real-time PCR, revealing a large difference in the expression level of MTase genes in different tissues. On the basis of their expression specificity MTase genes were divided into 3 basic groups (high, medium, and low expression). Each member of the first group (CMT1, MET1-1, DRM3, DRM1a, and $D R M 1 b$ ) was highly expressed in at least 1 specific tissue. The 2nd group included CMT2, DNMT2, MET1-2, and DRM2, which showed medium expression in almost all tissues. The 3rd group included a single gene, CMT3, which had low expression in all specific tissues. Judging by their expression patterns (Figure 2), it can be proposed that these genes may play roles in some basic metabolic pathways.

\section{Expression of cytosine DNA MTases with hormone treatment}

Because plant hormones play wide and important roles in all aspects of plant development and stress response, it is interesting to investigate the expression of cytosine DNA MTase family genes in response to plant hormones. Almost all genes were not regulated by diverse plant hormones including abscisic acid, gibberellin, auxin, brassinosteroid, cytokinin, and jasmonic acid (data not shown). These results indicate that cytosine DNA MTase family genes may not function under the regulation of plant hormones. There are only 2 cases, OsDRMIa and OsDRM1b, belonging to the $D R M$ subfamily, that were downregulated by jasmonic acid (Figure 3 ).

\section{Expression of cytosine DNA MTases in roots and shoots under stress conditions}

Spatial and temporal expression patterns of all DNA MTase genes of 4 subfamilies were observed in roots and shoots of 2-week-old seedlings. There was great variation in the expression activities of rice MTases under specific conditions of abiotic stress. Both in roots and shoots, our data did not deviate as reported previously (Teerawanichpan et al., 2009; Karan et al., 2012). The OsCMT1 gene was significantly downregulated $(\mathrm{P}<0.0001)$ in roots 

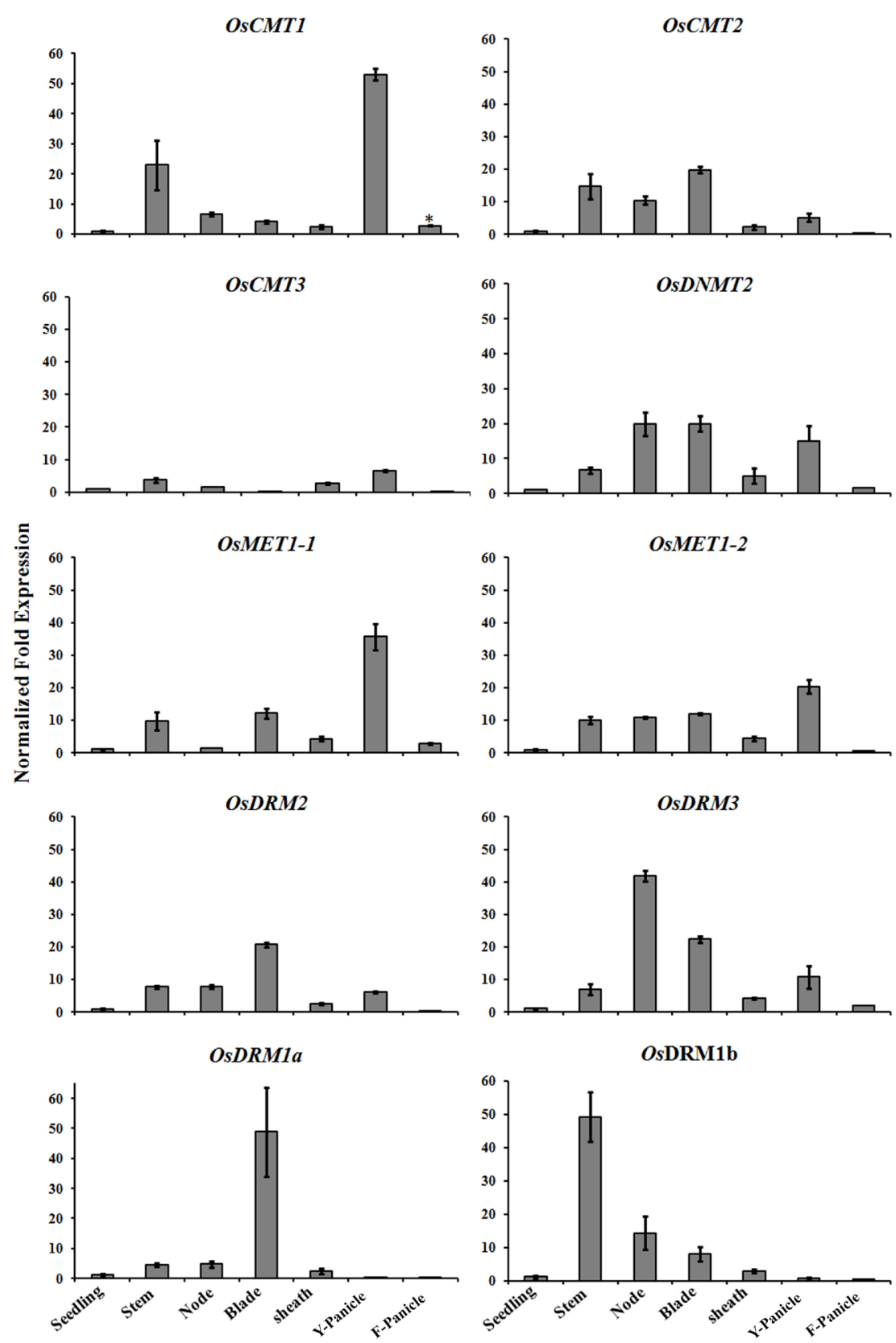

Figure 2. Expression profiles of the cytosine DNA MTase gene family in different tissues of rice based on quantitative real-time polymerase chain reaction (PCR). The rice plants were grown under natural conditions, and the samples of seedlings and different organs (stem, node, blade, sheath, y-panicle, and f-panicle) were collected at the booting stage. Y-panicle indicates the young (immature) panicle, while f-panicle indicates the flowering (matured) panicle. The bars report means $\pm \mathrm{SE}(\mathrm{N}=3)$. 
under both salt and osmotic stresses. In shoots, the $O S C M T 1$ gene also demonstrated a significant level of reduction in its expression $(\mathrm{P}<0.01)$. OsCMT2 showed no significant change in expression in roots under stress conditions, but it was highly upregulated $(\mathrm{P}<0.0001)$ in shoots under osmotic stress relative to the salt-stressed and control samples. The OsCMT3 gene was downregulated $(\mathrm{P}<0.0391)$ in roots under osmotic treatment as relative to saltstressed and control samples, while it was significantly upregulated $(\mathrm{P}<0.0049)$ in shoots under salt stress (Figure 4).
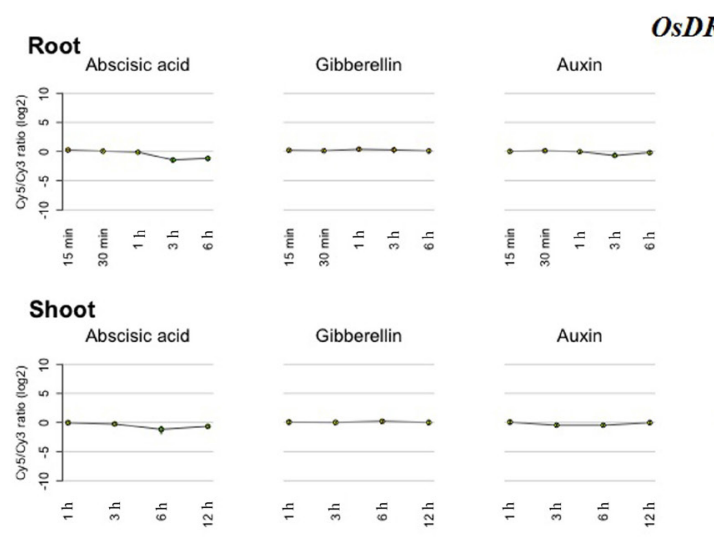

OsDRM1a
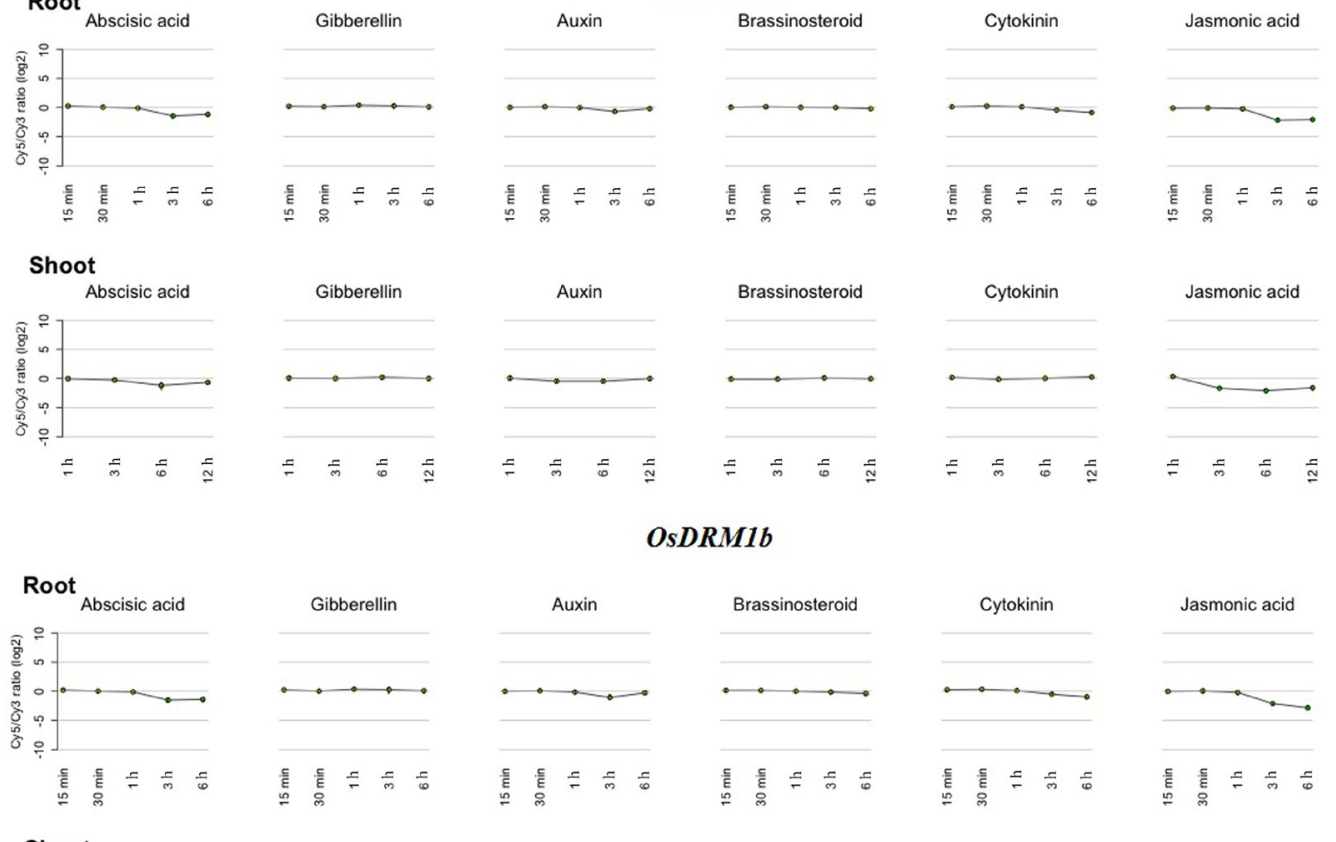

OsDRM1b
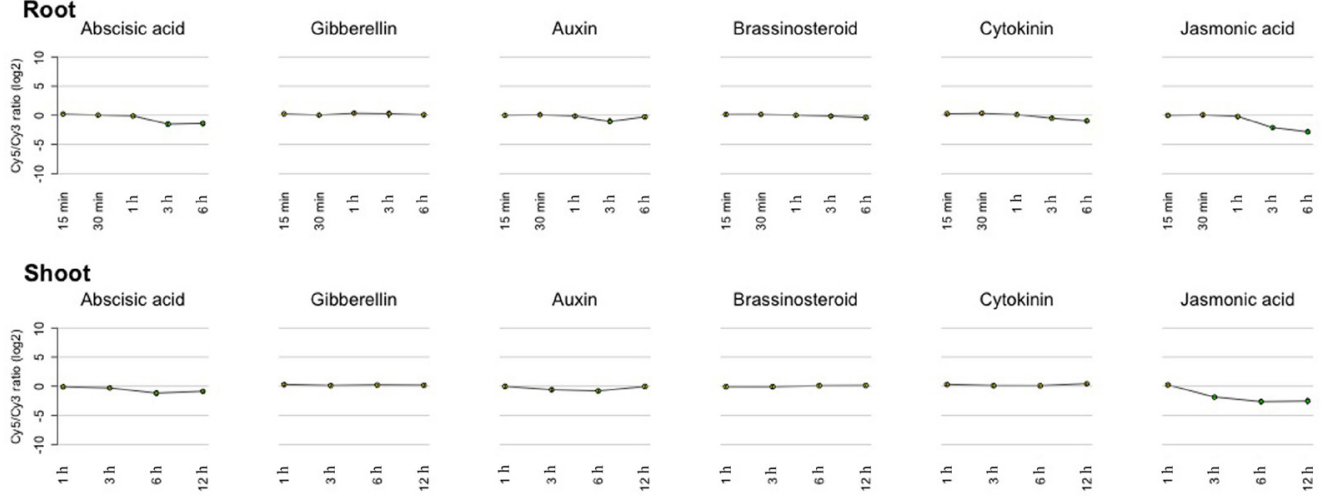

Figure 3. Expression patterns of OsDRMIa and OsDRMIb in roots and shoots of rice seedlings with hormone treatments. The expression analysis was conducted using the RiceXPro platform (http://ricexpro.dna.affrc.go.jp).

Statistically, OSDNMT2 expression was significantly upregulated $(\mathrm{P}<0.0001)$ in roots under salt stress compared to control, while its expression remained low under osmotic stress as compared to salt-stressed samples in roots. In shoots, OSDNMT2 was highly upregulated $(\mathrm{P}<0.0088)$ under salt treatment as compared to the other 2 conditions. OsMET1-1 expression was downregulated $(\mathrm{P}<0.0107)$ in roots of salt- and osmotic-stressed samples compared to control samples. In contrast, no significant difference in its expression was observed in shoots from the 3 conditions. Similarly, OsMET1-2 was also significantly downregulated $(\mathrm{P}<$ 
0.0168 ) in roots under osmotic stress, and no difference in expression was observed in shoots from control, salt-stressed, and osmotic-stressed samples (Figure 4); similar results were observed previously (Teerawanichpan et al., 2009) with respect to the differential expression of the MET1 family in rice.
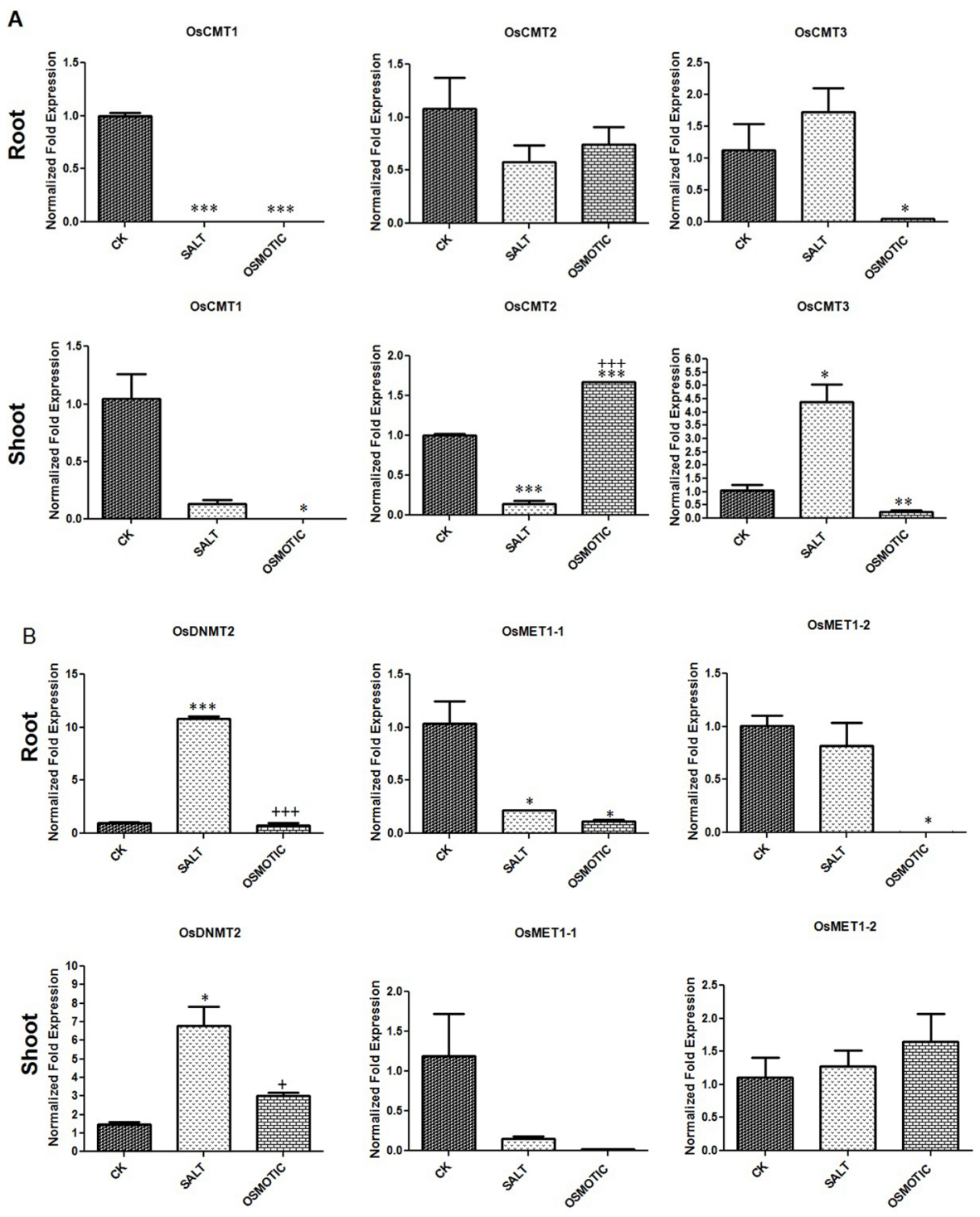

Figure 4. Expression patterns of 3 DNA cytosine MTase subfamilies in roots and shoots of rice seedlings under stress conditions. A. Expression of 3 genes (OsCMT1, OsCMT2, and OsCMT3) of the chromomethyltransferase subfamily. B. Expression of OSDNMT2 of the DNA methyltransferase 2 subfamily and 2 genes (OsMET1-1 and OsMET1-2) of the DNA methyltransferase 1 subfamily. $\mathrm{CK}=$ control. ${ }^{*,+} \mathrm{P}<0.01$; ${ }^{*}$ change as compared with the control, $(+)=$ the change in gene expression between salt and osmotic stress. The bars report means $\pm \mathrm{SE}(\mathrm{N}=3)$. 
Finally, the expression of 4 members of the DRM subfamily was calculated. OsDRMIa did not show any significant change in expression in roots, but it was highly upregulated $(\mathrm{P}<0.0012)$ in shoots under osmotic stress and salt stress compared to control. The OsDRM1b gene was downregulated in roots under osmotic stress, which is consistent with the result of a previous report (Moritoh et al., 2012). Fascinatingly, it was significantly upregulated $(\mathrm{P}<0.03704)$ in shoots under the same stressed condition as compared to salt treatment. The downregulation of OsDRM2 was observed $(\mathrm{P}<0.0005)$ under both stress conditions in root, and its expression was also low $(\mathrm{P}<0.0143)$ in shoots under osmotic stress. However, we did not observe significant levels of difference in the expression of OSDRM3 in root samples under the 3 conditions. Similarly, it was downregulated $(\mathrm{P}<0.0116)$ in shoots under osmotic stress (Figure 5).
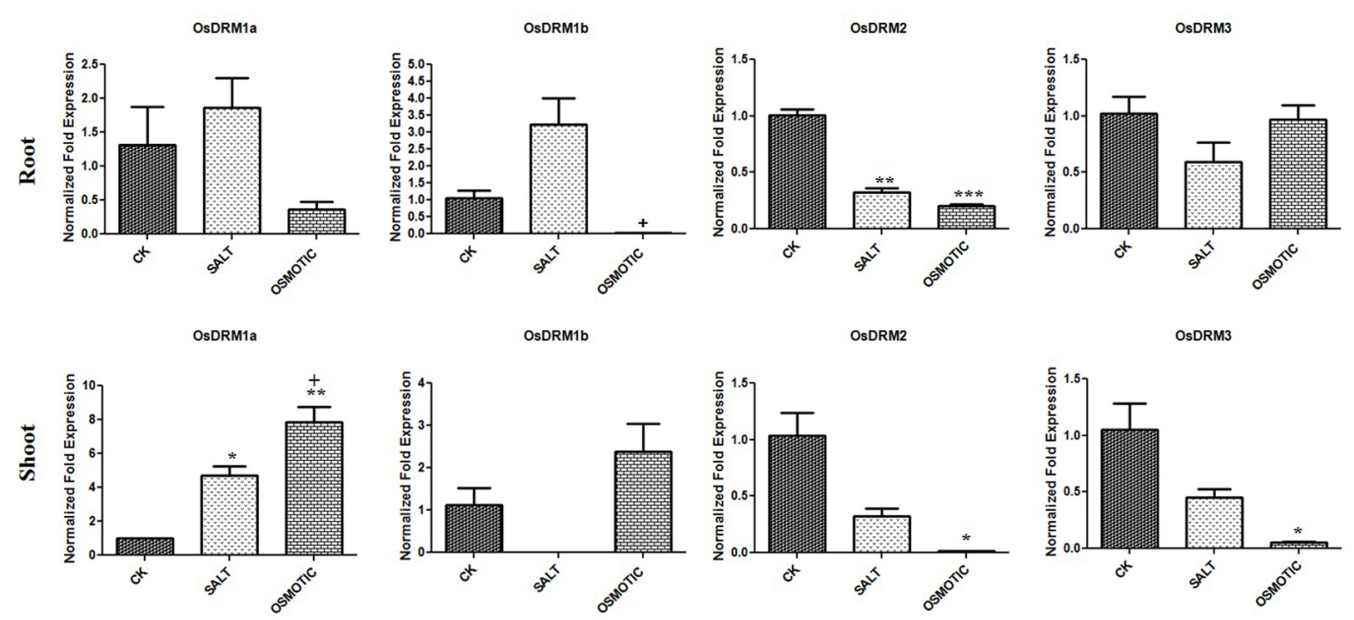

Figure 5. Expression patterns of the domains rearranged methyltransferase (DRM) subfamily of DNA MTases in roots and shoots of rice seedlings under stress conditions. The expression of 4 genes (OsDRMIa, OsDRM1b, OsDRM2, and OsDRM3) of the DRM subfamily of DNA MTases was analyzed by quantitative real-time PCR. Two-week-old seedlings were used after the application of the proper dose of both stresses for the proper time. CK $=$ control. ${ }^{*,+} \mathrm{P}<0.01 ;{ }^{*}$ change as compared with the control, $(+)=$ change in gene expression between salt and osmotic stress. The bars report means $\pm \mathrm{SE}(\mathrm{N}=3)$.

\section{Distinct expression of DNA MTases in 2 rice subspecies under stress conditions}

Japonica and indica are two major Asian rice subspecies that are distinguished by geographical and morphological bases. The cultivars of both subspecies demonstrate different levels of seed quality, seed maturity, and stress (abiotic and biotic) tolerance capacity (Liu et al., 2011). A comprehensive expression profile of all DNA MTase genes in 2 different cultivars was created. We examined the variation in the expression level of different MTases of the 2 cultivars under different conditions. In Nipponbare, there are some genes, like OsDRM1a and OsDRM1b, that were overexpressed under both stresses. However, the MTase expression levels in the indica cultivar (93-11) were opposite with salt and osmotic stress, as shown in Figure 6. 
A

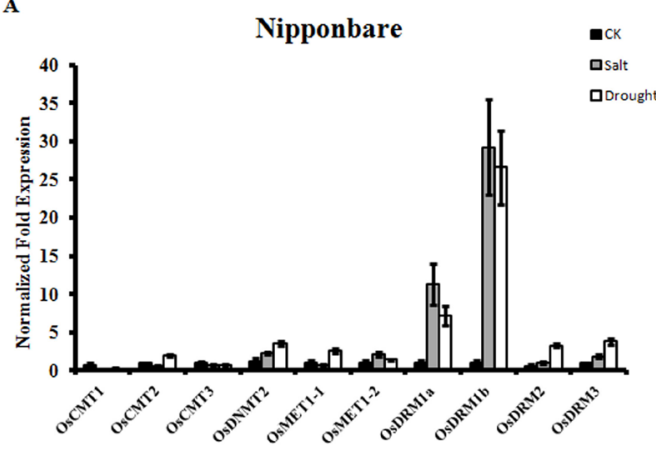

B

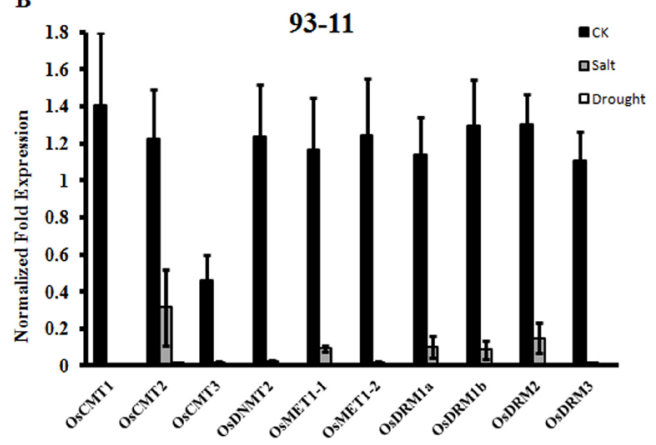

Figure 6. Expressional activities of all cytosine DNA MTases in Nipponbare (japonica) (A) and 93-11 (indica) (B) seedlings under salt and osmotic stresses. The mRNA level was calculated through quantitative real-time PCR. RNA was extracted from 2-week-old seedlings of control (CK), salt-stressed, and osmotic-stressed samples. The bars report means $\pm \mathrm{SE}(\mathrm{N}=3)$.

Finally, the 2 cultivars of the 2 subspecies showed great variation in the epigenetic regulation of environmental hazards. It is possible to analyze epigenetic mechanisms like DNA methylation, RNA-directed DNA methylation, and histone modification in 2 different subspecies of rice by comparing them.

\section{DISCUSSION}

Understanding the biological consequences of DNA methylation is a current focus of intensive research. Epigenetic modification induced by abiotic stresses (salt and osmotic) recovers to the original patterns once the stress is relieved. Some of these alterations may not recover to the original phase, which is called stress memory, and may be inherited through mitotic and even meiotic cell divisions (Chinnusamy and Zhu, 2009). Numerous reports and conclusions are uncovering the nature of DNA methylation regulation in plants against biotic and abiotic stresses, but many questions remain behind, including how plants sense the stresses and how the adaptive mechanisms are activated. Additionally, the action of various components in methylation pathways is also required to answer these questions. In eukaryotes, biotic and abiotic stresses may cause alterations in the level of DNA methylation (Chong and Whitelaw, 2004). DNA methylation patterns are considered to be important means of modifying the expression level of a number of genes (Boyko et al., 2007, 2010; Boyko and Kovalchuk, 2011). Thus, alterations in methylation patterns could be part of the plant protection mechanism in response to environmental perturbations. Kou et al. (2011) reported that, in rice, DNA methylation is involved in tolerance to nitrogen deficiency through the alteration of specific patterns. In this investigation, we analyzed the genome-wide expression profiles of cytosine DNA MTases in rice and observed their differential expression patterns under 2 different stress conditions. The DNA methylation status in the whole plant is regulated by various environmental, physiological, and developmental stress stimuli. The sources of DNA methylation (MTases) showed a significant level of difference in their expression with different environmental hazards compared to control conditions. For example, OsMET1-1 was highly downregulated in roots with both salt and osmotic stresses compared to control 
conditions. In addition, OsDNMT2 was significantly upregulated in roots with salt stress, while it was downregulated with osmotic stress. Therefore, the expression of rice MTases varied largely for genes and tissues with salt and osmotic stresses, indicating the complicated regulation that controls MTase expression in abiotic stress responses.

MTases are highly conserved in their structures, but their differential expression patterns suggested that they might play different biological roles in rice responses to these stresses. Moreover, based on our gene expression data from 2 rice subspecies, we concluded that DNA methylation adjustments may involve different levels of tolerance to abiotic stresses and that DNA methylation may play important roles in modulating plant tolerance. To further understand the biological significance of each DNA MTase in the rice response to abiotic stress, it is necessary to functionally analyze these genes using gene knockout mutants. This will help us to gain a better understanding of the epigenetic regulatory mechanisms of stress responses and will promote its application in genetic manipulation.

\section{ACKNOWLEDGMENTS}

Research supported by the National Science Foundation of China (\#31071069), the State Key Laboratory of Rice Biology (\#110101), and the "111" Project, and the Fundamental Research Funds for the Central Universities (\#KYZ201137).

\section{REFERENCES}

Arvidsson S, Kwasniewski M, Riano-Pachon DM and Mueller-Roeber B (2008). QuantPrime-a flexible tool for reliable high-throughput primer design for quantitative PCR. BMC Bioinformatics 9: 465.

Boyko A and Kovalchuk I (2011). Genome instability and epigenetic modification-heritable responses to environmental stress? Curr. Opin. Plant Biol. 14: 260-266.

Boyko A, Kathiria P, Zemp FJ, Yao Y, et al. (2007). Transgenerational changes in the genome stability and methylation in pathogen-infected plants: (virus-induced plant genome instability). Nucleic Acids Res. 35: 1714-1725.

Boyko A, Blevins T, Yao Y, Golubov A, et al. (2010). Transgenerational adaptation of Arabidopsis to stress requires DNA methylation and the function of Dicer-like proteins. PLoS One 5: e9514.

Cao X and Südhof TC (2001). A transcriptionally [correction of transcriptively] active complex of APP with Fe65 and histone acetyltransferase Tip60. Science 293: 115-120.

Chan SW, Henderson IR and Jacobsen SE (2005). Gardening the genome: DNA methylation in Arabidopsis thaliana. Nat. Rev. Genet. 6: 351-360.

Chan SW, Henderson IR, Zhang X, Shah G, et al. (2006). RNAi, DRD1, and histone methylation actively target developmentally important non-CG DNA methylation in Arabidopsis. PLoS Genet. 2: e83.

Chinnusamy V and Zhu JK (2009). Epigenetic regulation of stress responses in plants. Curr. Opin. Plant Biol. 12: 133-139.

Chong S and Whitelaw E (2004). Epigenetic germline inheritance. Curr. Opin. Genet. Dev. 14: 692-696.

Colomé-Tatché M, Cortijo S, Wardenaar R, Morgado L, et al. (2012). Features of the Arabidopsis recombination landscape resulting from the combined loss of sequence variation and DNA methylation. Proc. Natl. Acad. Sci. U. S. A. 109: 16240-16245.

Finnegan EJ and Kovac KA (2000). Plant DNA methyltransferases. Plant Mol. Biol. 43: 189-201.

Finnegan EJ, Peacock WJ and Dennis ES (1996). Reduced DNA methylation in Arabidopsis thaliana results in abnormal plant development. Proc. Natl. Acad. Sci. U. S. A. 93: 8449-8454.

Henikoff S and Comai L (1998). A DNA methyltransferase homolog with a chromodomain exists in multiple polymorphic forms in Arabidopsis. Genetics 149: 307-318.

Huang J, Wang MM, Jiang Y, Bao YM, et al. (2008). Expression analysis of rice A20/AN1-type zinc finger genes and characterization of ZFP177 that contributes to temperature stress tolerance. Gene 420: 135-144.

Jain M, Nijhawan A, Tyagi AK and Khurana JP (2006). Validation of housekeeping genes as internal control for studying gene expression in rice by quantitative real-time PCR. Biochem. Biophys Res. Commun. 345: 646-651. 
Karan R, DeLeon T, Biradar H and Subudhi PK (2012). Salt stress induced variation in DNA methylation pattern and its influence on gene expression in contrasting rice genotypes. PLoS One 7: e40203.

Kou HP, Li Y, Song XX, Ou XF, et al. (2011). Heritable alteration in DNA methylation induced by nitrogen-deficiency stress accompanies enhanced tolerance by progenies to the stress in rice (Oryza sativa L.). J. Plant Physiol. 168: 1685-1693.

Li E, Beard C and Jaenisch R (1993). Role for DNA methylation in genomic imprinting. Nature 366: 362-365.

Lindroth AM, Shultis D, Jasencakova Z, Fuchs J, et al. (2004). Dual histone H3 methylation marks at lysines 9 and 27 required for interaction with CHROMOMETHYLASE3. EMBO J. 23: 4286-4296.

Liu YC, Wu YR, Huang XH, Sun J, et al. (2011). AtPUB19, a U-box E3 ubiquitin ligase, negatively regulates abscisic acid and drought responses in Arabidopsis thaliana. Mol. Plant 4: 938-946.

Moritoh S, Eun CH, Ono A, Asao H, et al. (2012). Targeted disruption of an orthologue of DOMAINS REARRANGED METHYLASE 2, OsDRM2, impairs the growth of rice plants by abnormal DNA methylation. Plant J. 71: 85-98.

Ohba H, Steward N, Kawasaki S, Berberich T, et al. (2000). Diverse response of rice and maize genes encoding homologs of WPK4, an SNF1-related protein kinase from wheat, to light, nutrients, low temperature and cytokinins. Mol. Gen. Genet. 263: 359-366.

Pavlopoulou A and Kossida S (2009). Phylogenetic analysis of the eukaryotic RNA (cytosine-5)-methyltransferases. Genomics 93: 350-357.

Ponger L and Li WH (2005). Evolutionary diversification of DNA methyltransferases in eukaryotic genomes. Mol. Biol. Evol. 22: 1119-1128.

Rana RM, Dong S, Tang H, Ahmad F, et al. (2012). Functional analysis of OsHSBP1 and OsHSBP2 revealed their involvement in the heat shock response in rice (Oryza sativa L.). J. Exp. Bot. 63: 6003-6016.

Rausher MD (2001). Co-evolution and plant resistance to natural enemies. Nature 411: 857-864.

Reinders J, Wulff BB, Mirouze M, Marí-Ordóñez A, et al. (2009). Compromised stability of DNA methylation and transposon immobilization in mosaic Arabidopsis epigenomes. Genes Dev. 23: 939-950.

Rice JC, Briggs SD, Ueberheide B, Barber CM, et al. (2003). Histone methyltransferases direct different degrees of methylation to define distinct chromatin domains. Mol. Cell 12: 1591-1598.

Sharma R, Mohan Singh RK, Malik G, Deveshwar P, et al. (2009). Rice cytosine DNA methyltransferases - gene expression profiling during reproductive development and abiotic stress. FEBS J. 276: 6301-6311.

Sieńko M, NatorffR,ZielińskiZ, Hejduk A, etal.(2007). Two Aspergillus nidulans genes encoding methylenetetrahydrofolate reductases are up-regulated by homocysteine. Fungal Genet. Biol. 44: 691-700.

Tamura K, Peterson D, Peterson N, Stecher G, et al. (2011). MEGA5: molecular evolutionary genetics analysis using maximum likelihood, evolutionary distance, and maximum parsimony methods. Mol. Biol. Evol. 28: 2731-2739.

Teerawanichpan P, Krittanai P, Chauvatcharin N and Narangajavana J (2009). Purification and characterization of rice DNA methyltransferase. Plant Physiol. Biochem. 47: 671-680.

Teerawanichpan P, Chandrasekharan MB, Jiang Y, Narangajavana J, et al. (2004). Characterization of two rice DNA methyltransferase genes and RNAi-mediated reactivation of a silenced transgene in rice callus. Planta 218: 337-349.

Thompson JD, Gibson TJ, Plewniak F, Jeanmougin F, et al. (1997). The CLUSTAL X windows interface: flexible strategies for multiple sequence alignment aided by quality analysis tools. Nucleic Acids Res. 25: 4876-4882.

Xiao W, Custard KD, Brown RC, Lemmon BE, et al. (2006). DNA methylation is critical for Arabidopsis embryogenesis and seed viability. Plant Cell 18: 805-814.

Yamauchi T, Moritoh S, Johzuka-Hisatomi Y, Ono A, et al. (2008). Alternative splicing of the rice OsMET1 genes encoding maintenance DNA methyltransferase. J. Plant Physiol. 165: 1774-1782.

Yoshida S (1973). Effects of temperature on growth of the rice plant (Oryza sativa L.) in a controlled environment. Soil. Sci. Plant Nutr. 19: 299-310.

Zhu JK (2002). Salt and drought stress signal transduction in plants. Annu. Rev. Plant Biol. 53: 247-273. 